\title{
Utilidad de la determinación de la reserva ovárica como predictor de la posibilidad de embarazo espontáneo. Revisión sistemática
}

\author{
Gabriel Vallejos $P^{1}{ }^{1}$, Juan Enrique Schwarze M.MSc ${ }^{1,2}$, Sonia Villa V. ${ }^{2}$, Carolina Ortega \\ H. ${ }^{2}$, Ricardo Pommer T. 2 \\ ${ }^{1}$ Departamento Clínico de Obstetricia y Ginecología, Universidad de Santiago. ${ }^{2}$ Unidad Medicina Reproductiva Clínica \\ Monteblanco. Santiago, Chile.
}

\section{RESUMEN}

Antecedentes: En la actualidad, muchos especialistas determinan la reserva ovárica para aconsejar a sus pacientes acerca de su futuro reproductivo. Objetivo: Definir, a través de una revisión sistemática, si existe evidencia que justifique la determinación de la reserva ovárica como predictor de la posibilidad de embarazo espontáneo. Método: Realizamos una revisión sistemática usando las palabras claves "ovarian reserve" y "spontaneous pregnancy" en las bases MEDLINE y EMBASE, entre los años 2000 y 2015. Resultados: Sólo tres artículos cumplieron con los criterios de selección. Si bien difieren en la forma de determinar la reserva ovárica y la población analizada, ninguno de los estudios encontró que la determinación de la reserva ovárica tuviera utilidad clínica en predecir la posibilidad de un embarazo espontáneo. Conclusión: No existe evidencia que justifique la determinación de reserva ovárica, en forma rutinaria, para aconsejar a las parejas acerca de sus posibilidades de embarazo espontáneo.

\section{PALABRAS CLAVES: Reserva ovárica, embarazo espontáneo, hormona antimülleriana}

\section{SUMMARY}

Background: Many specialists use ovarian reserve tests to determine their patient's reproductive potential. Objective: To determine whether the ovarian reserve determine the possibility of spontaneous pregnancy. Method: We searched in MEDLINE and EMBASE, articles published between 2000 and 2015, with the keywords "spontaneous pregnancy" and "ovarian reserve". Results: Only three articles complied with the selection criteria. Although the studies have different approaches to evaluate ovarian reserve and study subjects, none of them found that that ovarian-reserve testing have clinical utility in predicting the chance of a spontaneous pregnancy. Conclusion: There is no utility to assess ovarian reserve routinely in order to predict chances of spontaneous pregnancy.

\section{KEY WORDS: Ovarian reserve, spontaneous pregnancy, anti-Müllerian hormone}

\section{INTRODUCCIÓN}

En Chile existe una tendencia a posponer la búsqueda del embarazo (1), lo que es un reflejo de la tendencia en países occidentales. Dado que las posibilidades de embarazo disminuyen con la edad de la mujer, esta tendencia ha llevado a un aumento en las parejas con posibles problemas de infertilidad (2). 
Para poder aportar a la toma de una decisión bien informada, es necesario estimar las posibilidades de embarazo de la pareja. Para lograr esto se han descrito modelos predictivos, que consideran especialmente la edad de la mujer, años de búsqueda de embarazo y motilidad espermática $(3,4)$.

Algunos profesionales han incorporado además la medición de la reserva ovárica, como otro de los factores productivos de embarazo. La reserva ovárica se refiere al número de folículos primordiales aún presentes en los ovarios (5). Es un importante factor pronóstico tanto para la respuesta ovárica a la hiperestimulación ovárica controlada como para el resultado del tratamiento en mujeres infértiles sometidas a fecundación in vitro (6-8). Los marcadores más utilizados para determinarla son: FSH plasmática, estradiol, inhibida $B$, hormona anti-Müllerina, determinación ultrasonográfica del número de foliculos antrales y del volumen ovárico, así como varios estudios dinámicos (i.e. test agonista Gnrh; test de provocación con citrato de clomifeno y el test de provocación externa con FSH) $(6,9)$.

El objetivo de esta revisión sistemática es determinar si existe evidencia que justifique la determinación de la reserva ovárica como predictor de la posibilidad de obtener embarazo espontáneo.

\section{MÉTODOS}

Se realizó un revisión sistemática de la literatura disponible en MEDLINE y EMBASE. Se buscaron los artículos que tuviesen los términos "ovarian reserve" y "spontaneous pregnancy" en del título o en el resumen.

Criterios de inclusión: estudios en inglés o español; publicados entre el 1 de enero de 2000 y 31 de julio de 2015; determinación de reserva ovárica antes del embarazo, o durante el primer trimestre; embarazo espontáneo.

Criterios de exclusión: estudios que incluyeran técnicas de reproducción asistida y estudios de tipo secundario. Dada la naturaleza de nuestra pregunta de investigación se aceptaron todos los diseños de estudios.

Basados en el título y resumen se escogieron los artículos que fueron revisados en extenso, y aplicados los criterios de selección por dos de los autores (GV y JES), independientemente. En caso de diferencias de opiniones, un tercer autor decidió si se incluía o no (SV o CO). Además, se revisó la bibliografía de estos estudios en búsqueda de nuevos trabajos.

Los datos relevantes de los artículos seleccionados fueron traspasados a una hoja de extracción previamente diseñada que incluia: título, autor principal, año de publicación, tipo de estudio, población estudiada, técnica de determinación de reserva ovárica, resultados y conclusiones de los autores.

\section{RESULTADOS}

La primera búsqueda arrojó 56 posibles artículos. Se buscó el trabajo completo, de acuerdo al título y resumen, al título y al resumen, en tres casos. Los tres artículos encontrados, cumplieron con los criterios de selección, y son presentados a continuación (Figura 1) (Tabla I).

Haadsma y cols (10), realizaron un estudio de cohorte prospectivo para determinar si el estudio de reserva ovárica agrega valor pronóstico a factores pronósticos previamente identificados por este grupo. La forma de determinar la reserva ovárica fue mediante recuento de folículos antrales, concentración de $\mathrm{FSH}$ e inhibina $\mathrm{B}$, y resultado del test de provocación con citrato de clomifeno. El modelo que utilizaban los autores incluía edad materna, duración de subfertilidad, tipo de subfertilidad (primaria o secundaria), motilidad espermática, y origen de la derivación médica. Para determinar la utilidad de los distintos estudios de reserva ovárica, los autores realizaron regresión de Cox. De las 474 pacientes que comenzaron el estudio, 75 pacientes lograron embarazo dentro del primer año. Los autores encontraron que, a pesar de que la combinación de FSH basal y el recuento de folículos antrales, mejoraba la capacidad predictiva de su modelo ya validado, la significancia clínica de estos hallazgos eran limitados. De hecho, ellos recomendaron no usarlos para predecir la posibilidad de embarazo espontáneo.

El grupo de Casedei y cols (11), realizó un estudio prospectivo en el que analizaron la proporción de embarazos espontáneos en 83 mujeres con diagnóstico de infertilidad sin causa aparente. Este diagnóstico fue hecho en base a ausencia de embarazo luego de un año sin anticoncepción, y sin alguna patología aparente. La reserva ovárica fue evaluada mediante la determinación de concentración plasmática de $\mathrm{AMH}$ y $\mathrm{FSH}$. Luego de seis meses de finalizado el estudio de infertilidad, catorce mujeres se habían embarazado. Los autores compararon la concentración de $\mathrm{AMH}$ y $\mathrm{FSH}$ con la de 69 mujeres que no lograron embarazo espontáneamente. Los resultados no demostraron diferencia estadísticamente significativa. La concentración media de $\mathrm{AMH}$ en mujeres con embarazo era 7,98 $\pm 6,37$ y en mujeres no embarazadas $13,47 \pm 10,94$ (no significativo); la concentración de FSH fue 9,9 $\pm 5,4$ y $8,1 \pm 3,9$ (no significativo), respectivamente.

El grupo de Streuli y cols (12), realizaron un estudio retrospectivo, en el que preguntaron a 87 mujeres puérperas acerca del tiempo necesario para conseguir embarazo, descartando los casos de tratamiento de infertilidad, y midieron los niveles de hormona anti-mülleriana en muestras de plasma congeladas en el primer trimestre. Encontraron que 16 mujeres tenían una concentración de $\mathrm{AMH}$ 
$<1,0 \mathrm{ng} / \mathrm{ml} ; 15$ tenían una concentración de 1,0$1,4 \mathrm{ng} / \mathrm{ml} ; 20$ tenían una concentración 1,5-1,9 ng/ $\mathrm{ml}$; y 36 tenían una concentración $\geq 2,0 \mathrm{ng} / \mathrm{ml}$. Los autores realizaron un análisis multivariado para determinar la posibilidad de conseguir embarazo en tres o seis meses. Para eso, consideraron edad, índice de masa corporal, hábito tabáquico, regularidad menstrual, frecuencia coital, paridad previa y concentración de AMH (<2ng/ml vs $\geq 2 \mathrm{ng} / \mathrm{ml}$ ). En ambos casos, la concentración de AMH no se asoció a un odds ratio significativo para posibilidad de embarazo. En el caso de embarazo en tres meses, el odds ratio fue 0,85 (IC 95\% 0,32-2,80), y en el caso de embarazo antes de seis meses el odds ratio fue $0,95(0,19-4,62)$, estadísticamente no significativo. En base a estos hallazgos, los autores concluyeron que los niveles de AMH no se asociaban con el tiempo necesario para conseguir el parto de un recién nacido vivo.

\section{DISCUSIÓN}

En esta revisión sistemática se incluye un total de tres artículos con un total 644 pacientes. Los tres autores coinciden en que la determinación de la reserva ovárica no tiene utilidad clínica a la hora de aconsejar a las pacientes que busquen embarazo espontáneo, a pesar de tener modelos distintos para responder esta pregunta.

Streuli y cols (12) realizaron su análisis en mujeres puérperas, en las que determinaron la concentración de AMH en muestras de plasma congeladas desde el primer trimestre de embarazo. Como una forma de determinar la fecundidad de la pareja, los autores le preguntaron a las parejas cuanto tiempo se habían demorado en conseguir embarazo, con todos los posibles sesgos de memoria asociados. Por otro lado, tanto Haadsma y cols (10), como Casadei y cols (11) asociaron el estudio de reserva

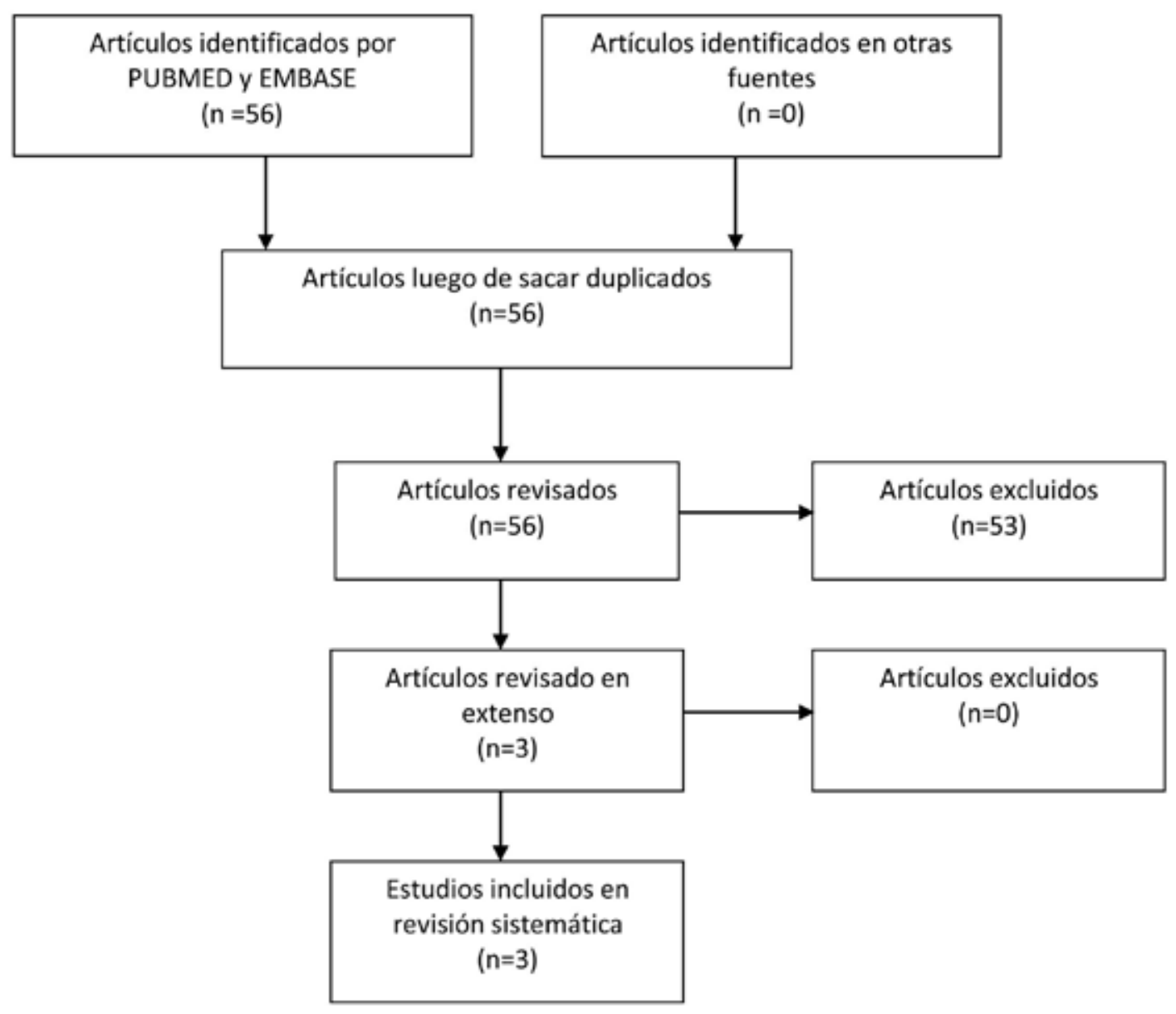

Figura 1. Selección de artículos para la revisión sistemática. 
ovárica con la posibilidad de embarazo espontáneo en parejas infértiles, sin causa aparente de infertilidad. El grupo de Haadsma ocupó los niveles de $\mathrm{FSH}$, recuento de folículos antrales y otros, para determinar si estas variables agregan poder estadístico a su modelo ya validado, lo que tiene escasa utilidad clínica. El grupo de Casadei ocupó los niveles de $\mathrm{AMH}$, sin encontrar diferencias en las media en el grupo que logró embarazo y el grupo que no logró; hay que destacar, que su estudio no tuvo un cálculo de tamaño muestral para tener un poder estadístico suficiente para demostrar alguna diferencia.

Ninguno de los estudios analizados tiene un modelo adecuado para responder la pregunta acerca de la utilidad de la determinación de reserva ovárica para predecir la posibilidad de embarazo en forma prospectiva en parejas no infértiles.

Tabla I

RESUMEN DE LOS ARTÍCULOS REVISADOS

\begin{tabular}{|c|c|}
\hline Autor, año, (cita) & Población \\
\hline Haadasma, 2008 (10) & $\begin{array}{l}\text { Estudio prospectivo de } 474 \\
\text { mujeres con diagnóstico } \\
\text { de infertilidad de causa } \\
\text { desconocida que fueron } \\
\text { evaluadas a los doce } \\
\text { meses }\end{array}$ \\
\hline
\end{tabular}

Casadei, 2013 (11)

Estudio prospectivo de 83 mujeres con infertilidad inexplicada y con reserva ovárica normal o baja.
Estudio retrospectivo de 87 mujeres que planearon embarazo y resultó en un recién nacido vivo, a las que se les preguntó por el tiempo necesario para conseguir embarazo.
Hormona anti-Mülleriana al momento del estudio

Hormona anti-Mülleriana en el primer trimestre del embarazo
75 parejas (15,8\%) lograron embarazo espontáneo, con recién nacido vivo. No se encontraron valores predictivos significativos entre los diferentes métodos de medición de reserva ovárica. Los autores no recomiendas el uso de medición de reserva ovárica rutinaria para predecir embarazo espontáneo

No hubo diferencias significativas en los valores medidos de hormona anti-Mülleriana entre mujeres embarazadas y aquellas no embarazadas. De las 14 pacientes que lograron embarazo espontáneo, 5 tenían valores de hormona anti-Mülleriana < a 0,4 ng/dL, por lo que no se encuentra relación de la hormona anti-Mülleriana para predicción de embarazo espontáneo. Incluso con niveles bajos de hormona anti-Mülleriana puede ocurrir embarazo

$55,9 \%$ de los embarazos fue durante los primeros 3 meses de exposición, $82 \%$ a los 6 meses y $88,1 \%$ dentro de un año. Se dividió en valores de hormona anti-Mülleriana $<2,0 \mathrm{ng} / \mathrm{dL}$ y $>2,0 \mathrm{ng} / \mathrm{dL}$. Se encontró que la hormona anti-Mülleriana no se asocia significativamente con el tiempo efectivo de embarazo, independiente de los niveles de hormona anti-Mülleriana. 


\section{CONCLUSIÓN}

La evidencia encontrada no justifica la determinación de reserva ovárica para predecir la posibilidad de embarazo en parejas no infértiles y en parejas infértiles sin causa aparente de su infertilidad.

\section{REFERENCIAS}

1. Fuentes A, Jesam C, Devoto L, Angarita B, GalleguiIlos A, Torres A, et al. Association between motherhood postponement and socioeconomic sta-tus. Rev Med Chil 2010;138(10):1240-5.

2. te Velde ER, Pearson PL. The variability of female reproductive ageing. Hum Reprod Update 2002;8(2):141-54.

3. Eimers JM, te Velde ER, Gerritse R, Vogelzang ET, Looman CW, Habbema JD. The prediction of the chance to conceive in subfertile cou-ples. Fertil Steril 1994;61(1):44-52.

4. Collins JA. Overall prognosis with current treatment of infertility. Hum Reprod Update 2004;10(4):309-16.

5. Faddy MJ, Gosden RG. A mathematical model of follicle dynamics in the human ovary. Hum Reprod 1995;10(4):770-5.

6. Broekmans FJ, Kwee J, Hendriks DJ, Mol BW, Lambalk $C B$. A systematic review of tests predicting ovarian reserve and IVF outcome. Hum Reprod Update 2006;12(6):685-718.
7. La Marca A, Sunkara SK. Individualization of controlled ovarian stimulation in IVF using ovarian reserve markers: from theory to practice. Hum Reprod Update 2013;20(1):124-40.

8. Streuli I, Fraisse T, Chapron C, Bijaoui G, Bischof $P$, de Ziegler D. Clinical uses of anti-Müllerian hormone assays: pitfalls and promises. Fertil Steril 2009;91(1):226-30.

9. Bukulmez $O$, Arici A. Assessment of ovarian reserve. Curr Opin Obstet Gynecol 2004;16(3):231-7.

10. Haadsma ML, Groen H, Fidler V, Bukman A, Roeloffzen EMA, Groenewoud ER, et al. The predictive value of ovarian reserve tests for spontaneous pregnancy in subfertile ovulatory women. Hum Reprod Ox-ford University Press 2008;23(8):1800-7.

11. Casadei L, Manicuti C, Puca F, Madrigale A, Emidi $\mathrm{E}$, Piccione E. Can anti-Müllerian hormone be predictive of spontaneous onset of pregnancy in women with unexplained infertility? J Obstet Gynaecol 2013;33(8):857-61.

12. Streuli I, de Mouzon J, Paccolat C, Chapron C, Petignat $\mathrm{P}$, Irion $\mathrm{OP}$, et al. AMH concentration is not related to effective time to pregnancy in women who conceive naturally. Reprod Biomed Online 2014;28(2):216-24. 